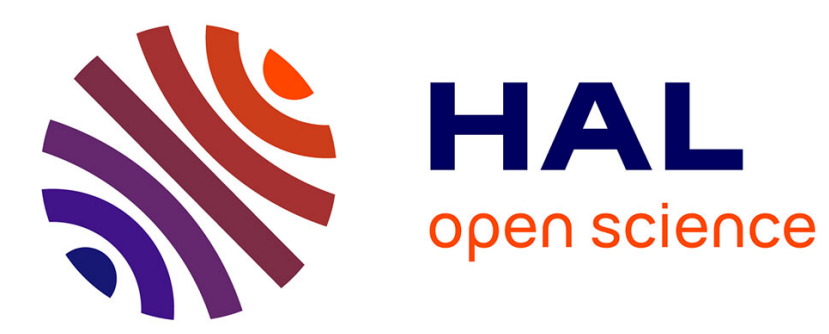

\title{
Coherent anti-Stokes Raman Fourier ptychography
}

Sandro Heuke, Kevin Unger, Samira Khadir, Kamal Belkebir, Patrick C.

Chaumet, Herve Rigneault, Anne Sentenac

\section{To cite this version:}

Sandro Heuke, Kevin Unger, Samira Khadir, Kamal Belkebir, Patrick C. Chaumet, et al.. Coherent anti-Stokes Raman Fourier ptychography. Optics Express, 2019, 27 (16), pp.23497. 10.1364/OE.27.023497 . hal-02266917

\section{HAL Id: hal-02266917 \\ https://hal.science/hal-02266917}

Submitted on 24 Nov 2019

HAL is a multi-disciplinary open access archive for the deposit and dissemination of scientific research documents, whether they are published or not. The documents may come from teaching and research institutions in France or abroad, or from public or private research centers.
L'archive ouverte pluridisciplinaire HAL, est destinée au dépôt et à la diffusion de documents scientifiques de niveau recherche, publiés ou non, émanant des établissements d'enseignement et de recherche français ou étrangers, des laboratoires publics ou privés. 


\title{
Coherent anti-Stokes Raman Fourier ptychography
}

\author{
Sandro Heuke, ${ }^{1}$ Kevin Unger, ${ }^{1}$ Samira Khadir, ${ }^{1}$ Kamal \\ Belkebir, ${ }^{1}$ Patrick C. Chaumet, ${ }^{1}$ Hervé Rigneault, ${ }^{1,2}$ And \\ Anne SentenAC ${ }^{1,3}$ \\ ${ }^{1}$ Aix Marseille Univ, CNRS, Centrale Marseille, Institut Fresnel, Marseille, France \\ ${ }^{2}$ herve.rigneault@fresnel.fr \\ 3 anne.sentenac@fresnel.fr
}

\begin{abstract}
We present a theoretical and numerical study of coherent anti-Stokes Raman scattering Fourier ptychography microscopy (CARS-FPM), a scheme that has not been considered so far in the previously reported CARS wide-field imaging schemes. In this approach, the distribution of the Raman scatterer density of the sample is reconstructed numerically from CARS images obtained under various angles of incidences of the pump or Stokes beam. Our inversion procedure is based on an accurate vectorial model linking the CARS image to the sample and yields both the real and imaginary parts of the susceptibility, the latter giving access to the Raman information, with an improved resolution.
\end{abstract}

(C) 2019 Optical Society of America under the terms of the OSA Open Access Publishing Agreement

\section{Introduction}

Coherent anti-Stokes Raman (CARS) microscopy is a marker-free imaging tool in which the sample is illuminated simultaneously by two intense beams at different pump and Stokes wavelengths and emits an anti-stokes radiation resulting from a non-linear light-matter interaction [1-3]. This technique permits to probe the third-order susceptibility of the sample which is highly dependent on the chemical components of the material. As a result, CARS microscopy is able to provide chemical images of biological samples $[4,5]$ and more recently opened interesting perspectives for histo-pathological applications [6-9]. Yet, to appear as a breakthrough in the bio-medical investigation of tissues, CARS microscopy has still to demonstrate its ability to yield highly resolved quantitative images with a reasonable acquisition rate. Because of the high power involved, CARS microscopy has been mostly implemented with focused lasers scanning the sample [1]. This point-wise approach proved to be particularly interesting for 3D sectioning of thick samples down to hundreds of micrometer of depth due to its non-linearity providing an intrinsic confocality property. On the other hand, for thin samples, i.e. with an axial extent of only a few micrometers (e.g. $<10 \mu \mathrm{m})$, the confocality loses its relevance and the scanning technique appears orders of magnitude too slow to compete with the traditional widefield imaging of stained tissues. Hence, in the last fifteen years, several widefield configurations based on different illumination geometries for the pump and Stokes beam, such as co-propagating [10-13], folded-box [11,14-18], oblique [19,20], converging beam [21] or light grids [22], have been investigated in combination with holography [23] or Fourier-transform spectroscopy [24].

Surprisingly, none of these wide field studies developed a model linking the CARS image to the sample susceptibility, thus preventing any comparison in terms of resolution between the different configurations. In this work, we derive the relationship between the sample and any CARS image obtained under widefield excitation and we analyze the resolution that can be expected with a given illumination and observation configuration. Then, we investigate a novel wide-field CARS image acquisition paradigm: CARS Fourier ptychography microscopy (CARS FPM) in which various low resolved images recorded for different illumination angles of the pump or Stokes beam are processed numerically to yield the sample's complex third-order susceptibility with 
high resolution [25]. This work paves the way towards highly resolved quantitative widefield CARS microscopy.

The paper is divided in three main parts. First we study the anti-Stokes radiation emitted by the sample in far-field and we link the notion of transfer function to the phase matching conditions. Second, we develop a vectorial model of the widefield CARS image of a microscope in a $4 f$ configuration. Based on this model, we develop an inversion procedure able to reconstruct the sample density from several CARS images recorded for various excitations. The potential of CARS FPM is then tested on synthetic data. Last, we present a possible experimental setup with a particular attention to the requirements of a suitable laser source.

\section{Analysis of the transfer function and phase matching conditions}

In the literature, the concepts of phase-matching conditions and spatial frequency transfer functions are frequently used but by non-overlapping scientific communities due to a lack of mutual understanding. In this section, we show the relationship between these two approaches. Hereafter, the subscripts $S, p$ and $a S$ indicate affiliations to the Stokes, pump beam and anti-Stokes radiation, respectively.

The phase matching conditions tell us that, if a homogeneous non-linear infinite medium is excited by a pump and Stokes collimated beams $\mathbf{E}_{p, S}$ of angular frequency $\omega_{p, S}$ respectively and wavevectors $\mathbf{k}_{p, S}$, then it generates an anti-Stokes beam with angular frequency $\omega_{a S}=2 \omega_{p}-\omega_{S}$ and wavevector

$$
\mathbf{k}_{a S}=2 \mathbf{k}_{p}-\mathbf{k}_{S} .
$$

In CARS imaging, one seeks to retrieve the varying sample third order susceptibility from the CARS radiation. The first issue is thus to analyse how the phase matching conditions are modified when the sample is inhomogeneous.

In the following, we assume that the sample placed in an homogeneous medium with refractive index $n_{p, S, a S}$, excited by two linear polarized plane waves $\mathbf{E}_{p, S}$ at the pump and Stokes frequencies generates an anti-Stokes radiation at $\omega_{a S}=2 \omega_{p}-\omega_{S}$. The wavevectors of the pump, stokes and anti-stokes fields satisfy, $k_{p, S, a S}=n_{p, S, a S} \omega_{p, S, a S} / c$ respectively.

The source of anti-Stokes radiation is linked to the third-order polarization defined as [26]:

$$
\mathbf{P}_{a S}^{(3)}(\mathbf{r})=\overleftrightarrow{\chi}_{a S}^{(3)}(\mathbf{r}) \mathbf{E}_{p}(\mathbf{r}) \mathbf{E}_{p}(\mathbf{r}) \mathbf{E}_{S}^{*}(\mathbf{r})
$$

which obeys, in the slowly varying envelop approximation, the equation,

$$
\boldsymbol{\nabla} \times \boldsymbol{\nabla} \times \mathbf{E}_{a S}(\mathbf{r})-k_{a S}^{2} \mathbf{E}_{a S}(\mathbf{r})=k_{a S}^{2} \mathbf{P}_{a S}^{(3)}(\mathbf{r}),
$$

where $\overleftrightarrow{\chi}_{a S}^{(3)}(\mathbf{r})$ denotes the third order nonlinear susceptibility tensor of rank 4 which is a function of the dipole or scatterer density $N(\mathbf{r})$, its orientation and the Raman scattering cross section [2]. Each of its 81 tensor elements may contribute to the anti-Stokes polarization with polarization orientation $A$ using Einstein notation as $P_{a S, A}^{(3)}=\chi_{a S, A B C D}^{(3)} E_{p, B} E_{p, C} E_{S, D}^{*}$ with $A, B, C, D \in x, y, z$. Introducing the Green tensor which is the solution of

$$
\boldsymbol{\nabla} \times \boldsymbol{\nabla} \times \overleftrightarrow{\mathbf{G}}(\mathbf{R}, \mathbf{r})-k_{a S}^{2} \overleftrightarrow{\mathbf{G}}(\mathbf{R}, \mathbf{r})=\overleftrightarrow{\mathbf{I}} \delta(\mathbf{R}-\mathbf{r})
$$

that satisfies out-going boundary condition, the anti-Stokes radiation at observation point $\mathbf{R}$ is given by [26]

$$
\mathbf{E}_{a S}(\mathbf{R})=k_{a S}^{2} \int \mathrm{d} \mathbf{r} \overleftrightarrow{\mathbf{G}}(\mathbf{R}, \mathbf{r}) \mathbf{P}_{a S}^{(3)}(\mathbf{r})
$$

The link between the anti-Stokes radiation and the sample is easily obtained when the observation point is far enough (Fraunhofer diffraction regime) from the sample for the radiated field to be 
approximated by a centered spherical wave whose amplitude depends only on the direction of observation $\mathbf{k}_{a S}=k_{a S} \mathbf{R}_{\text {far }} / R_{\text {far }}$. The far-field amplitude, $\mathbf{e}\left(\mathbf{k}_{a S}\right)$, can be directly recorded at the Fourier or pupil plane of a microscope [27], each point of the latter corresponding to a specific direction of observation. In far-field, the Green tensor can be approximated by,

$$
\overleftrightarrow{\mathbf{G}}\left(\mathbf{R}_{\mathrm{far}}, \mathbf{r}\right) \approx \overleftrightarrow{\mathbf{M}}\left(\mathbf{k}_{a S}\right) \exp \left(-i \mathbf{k}_{a S} \cdot \mathbf{r}\right) /\left(4 \pi R_{\mathrm{far}}\right)
$$

with $\overleftrightarrow{\mathbf{M}}\left(\mathbf{k}_{a S}\right)$ defined as,

$$
\overleftrightarrow{\mathbf{M}}\left(\mathbf{k}_{a S}\right) \mathbf{P}=\mathbf{P}-\left[\mathbf{P} \cdot \mathbf{k}_{a S}\right] \mathbf{k}_{a S} / k_{\mathrm{aS}}^{2}
$$

is the projection operator onto the plane normal to $\mathbf{k}_{a S}[26,28]$ and $\mathrm{P}$ a generic polarization vector. Injecting Eq. (6) in Eq. (5), one obtains the expression for the CARS far-field,

$$
\mathbf{E}_{a S}\left(\mathbf{R}_{\mathrm{far}}\right) \approx \frac{2 \pi^{2} k_{a S}^{2} \exp \left(i k_{\mathrm{aS}} R_{\mathrm{far}}\right)}{R_{\mathrm{far}}} \mathbf{e}_{a S}\left(\mathbf{k}_{a S}\right),
$$

with

$$
\mathbf{e}_{a S}\left(\mathbf{k}_{a S}\right)=\overleftrightarrow{\mathbf{M}}\left(\mathbf{k}_{a S}\right) \tilde{\mathbf{P}}_{a S}^{(3)}\left(\mathbf{k}_{a S}\right)
$$

where we have introduced the 3D Fourier transform of $\mathbf{P}^{(3)}$,

$$
\mathcal{F}_{3 D}(f)=\tilde{f}(\mathbf{k})=\frac{1}{8 \pi^{3}} \int f(\mathbf{r}) e^{-i \mathbf{k} \cdot \mathbf{r}} \mathrm{d} \mathbf{r} \text { and } \mathcal{F}_{3 D}^{-1}(\tilde{f})=\int \tilde{f}(\mathbf{k}) e^{i \mathbf{k} \cdot \mathbf{r}} \mathrm{d} \mathbf{k} .
$$

To simplify our analysis, we will assume hereafter that the susceptibility tensor is proportional to the scatterer density

$$
\overleftrightarrow{\chi}_{a S}^{(3)}(\mathbf{r})=N(\mathbf{r}) \overleftrightarrow{\chi}_{a S}^{\prime(3)}
$$

with $\overleftrightarrow{\chi}_{a S}^{\prime(3)}$ known. The density $N(\mathbf{r})=N_{e l}(\mathbf{r})+N_{r e, v i b}(\mathbf{r})+i N_{i m, v i b}(\mathbf{r})$ is complex, where $N_{e l}(\mathbf{r})$ denotes the electronic contribution giving rise to the nonresonant background. $N_{r e, v i b}(\mathbf{r})+$ $i N_{i m, v i b}(\mathbf{r})$ express the vibrational contribution. It should be noted that $N_{r e, v i b}(\mathbf{r})$ is spatially independent from $N_{i m, v i b}(\mathbf{r})$ as soon as the nonhomogeneous sample consists of several molecular groups of unequal Raman shifts that contribute to the overall CARS signal.

Recalling that the excitation beams are plane waves, $\mathbf{E}_{p, S}(\mathbf{r})=\exp \left(i \mathbf{k}_{p, S} \cdot \mathbf{r}\right) \mathbf{u}_{p, S}$, the third order polarization can be written as,

$$
\mathbf{P}_{a S}^{(3)}(\mathbf{r})=N(\mathbf{r}) \exp \left[i\left(2 \mathbf{k}_{p}-\mathbf{k}_{S}\right) \cdot \mathbf{r}\right] \mathbf{u}
$$

where $\mathbf{u}=\overleftrightarrow{\chi}_{a S}^{\prime(3)} \mathbf{u}_{p} \mathbf{u}_{p} \mathbf{u}_{S}^{*}$. Using the modulation theorem and Eq. (12), the 3D Fourier transform of the polarization verifies,

$$
\tilde{\mathbf{P}}_{a S}^{(3)}(\mathbf{k})=\tilde{N}\left(\mathbf{k}-2 \mathbf{k}_{p}+\mathbf{k}_{S}\right) \mathbf{u},
$$

and the far-field CARS amplitude is,

$$
\mathbf{e}_{a S}\left(\mathbf{k}_{a S}\right) \propto \tilde{N}\left(\mathbf{k}_{a S}-2 \mathbf{k}_{p}+\mathbf{k}_{S}\right) \overleftrightarrow{\mathbf{M}}\left(\mathbf{k}_{a S}\right) \mathbf{u}
$$

Thus, the recording of the anti-Stokes far-field amplitude at a point of the microscope pupil plane corresponding to the observation direction $\mathbf{k}_{a S}$ depends on the three-dimensional Fourier coefficient of the scatterer density $N$ taken at $\mathbf{K}=\mathbf{k}_{a S}-2 \mathbf{k}_{p}+\mathbf{k}_{S}$. Now, if the non-linear medium is homogeneous and infinite, $\tilde{N}(\mathbf{K})=0$ everywhere except for $\mathbf{K}=\mathbf{0}$. In this case, the CARS radiation occurs only in the direction $\mathbf{k}_{a S}$ such that $\mathbf{k}_{a S}=2 \mathbf{k}_{p}-\mathbf{k}_{S}$. The classical phase 
matching condition is retrieved. On the contrary, when the sample is inhomogeneous, the CARS field is emitted in all the directions,

$$
\mathbf{k}_{a S}=\mathbf{K}+2 \mathbf{k}_{p}-\mathbf{k}_{s}
$$

for which $\tilde{N}(\mathbf{K})$ is not negligible.

In an imaging experiment, one uses the CARS radiation to retrieve the scatterer density. The one to one relationship, Eq. (14), between the far-field CARS amplitude and the Fourier coefficient of the scatterer density gives access to the scatterer density spectrum in a restricted three-dimensional Fourier domain, hereafter named $W$, that depends on the imaging configuration. The domain $W$ is built by following the extremity of the transfer vector $\mathbf{K}=\mathbf{k}_{a S}-2 \mathbf{k}_{p}+\mathbf{k}_{S}$ when $\mathbf{k}_{p, S}$, fixed by the pump and Stokes beam, and $\mathbf{k}_{a S}$ corresponding to the observation directions, are varied. This support determines the resolution with which the scatterer density can be reconstructed.

In an ideal widefield CARS imaging set-up, where the far-field radiation is observed from all possible angles (using a $4 \pi$ objective for example [29]), while the pump and Stokes beams are fixed, $W$ is a sphere of radius $k_{a S}$ translated by $2 \mathbf{k}_{p}-\mathbf{k}_{S}$. In an ideal widefield CARS tomography, where the observation and illumination are done from all possible directions, $\Omega$ is a ball of radius $k_{a S}+2 k_{p}+k_{S}$. As a reminder, the Fourier support of an ideal linear tomography experiment at the pump wavelength is a ball of radius $2 k_{p}$ [30]. Thus, CARS has the potential to provide three-dimensional images with a twice better resolution than that of an ideal linear tomography set-up, which would be about $\lambda_{p} / 8$.

Yet, generally the observation and illumination directions are restricted by the microscope configuration. In particular, the observation directions are limited by the size of the pupil of the microscope objective. Introducing the transverse and axial components of the wavevector, $\mathbf{k}_{a S}=\mathbf{k}_{\|}+\gamma \mathbf{z}$ where $\gamma=\sqrt{k_{a S}^{2}-k_{\|}^{2}}$ and $\mathbf{z}$ is the unit vector along the optical axis, the pupil imposes that $k_{\|}<k_{a S}$ NA where NA is the numerical aperture of the objective. As a result, $\mathbf{k}_{a S}$ describes a cap of sphere of radius $k_{a S}$ whose projection onto the transverse $\left(k_{x}, k_{y}\right)$ plane is a disk of radius of $k_{a s} \mathrm{NA}$.

Figure 1 illustrates Eq. (15). In most experiments, the momentum transfer between the axial components of the wavevectors is assumed to be always fulfilled so that only the transverse components are accounted for in Eq. (15). In particular, this condition is satisfied for thin samples (axial extension $<\lambda_{a S}$ ).

We conclude this section with a comment on folded box illumination configurations [11,14-17]. From Fig. 1 as well as Eq. (14), it is evident that a simultaneous illumination with two pump (probe) beams with wavevectors $\mathbf{k}_{p 1}$ and $\mathbf{k}_{p 2}$ featuring different incident angles leads to mixing terms in the far-field amplitude so that $\mathbf{e}\left(\mathbf{k}_{a S}\right)$ is now related to $2 \tilde{N}\left(\mathbf{k}_{a S}+\mathbf{k}_{p 1}+\mathbf{k}_{p 2}-\mathbf{k}_{S}\right)+$ $\tilde{N}\left(\mathbf{k}_{a S}+\mathbf{k}_{p 1}+\mathbf{k}_{p 1}-\mathbf{k}_{S}\right)+\tilde{N}\left(\mathbf{k}_{a S}+\mathbf{k}_{p 2}+\mathbf{k}_{p 2}-\mathbf{k}_{S}\right)$. Thus, the image will be composed of the coherent superposition of these three band-passes which may results in artifacts if the absolute values of sample spectrum are not sufficiently low for high spatial frequencies. We now focus on one possible technique for retrieving the sample spectrum on a large Fourier domain, CARS Fourier ptychography microscopy. To this aim, we first derive a comprehensive vectorial model of the CARS images obtained in a wide-field microscope which will be the basis of the reconstruction procedure.

\section{Modeling the CARS image of a widefield microscope}

We consider an imaging configuration similar to that of the previous section, see Fig. 5, in which the sample is excited by a pump and Stokes beams that are approximated by plane waves. The CARS radiation is recorded at the image plane of the microscope by propagating through an objective (L1) with focal $f_{1}$ and numerical aperture NA and a tube lens (L2) with focal $f_{2}$ placed 


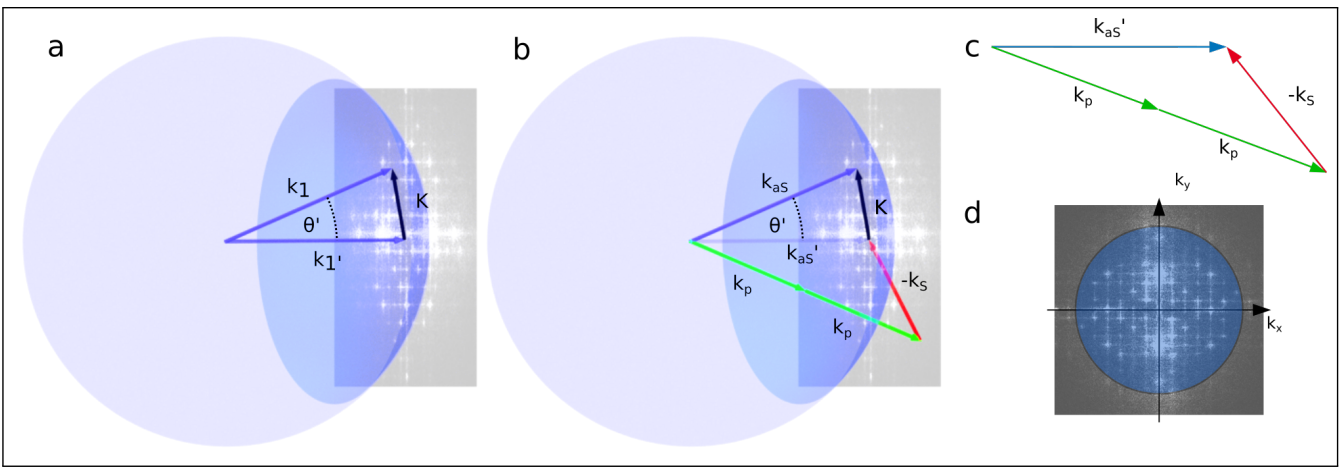

Fig. 1. Phase-matching and lens transfer: a) Linear scattering: the incident plane wave of wave vector $\mathbf{k}_{1}^{\prime}$ is scattered into direction $\mathbf{k}_{1}$ if the object contains the spatial frequency vector $\mathbf{K}$ following $\mathbf{k}_{1}^{\prime}+\mathbf{K}=\mathbf{k}_{1}$. The scattered wave is collected through the pupil of the microscope if its transverse component, $\mathbf{k}_{\|, 1}$ satisfies, $k_{\|, 1} \leq k_{1} \mathrm{NA}$, i.e. if $\mathbf{k}_{1}$ ends on the cap of the sphere outlined in dark blue. b) Nonlinear four-wave-mixing: the effective incident wave vector in direction $\mathbf{k}_{a S}^{\prime}=\mathbf{k}_{p}+\mathbf{k}_{p}-\mathbf{k}_{S}$ for an homogeneous infinite nonlinear medium is scattered in direction $\mathbf{k}_{a S}=\mathbf{k}_{a S}^{\prime}+\mathbf{K}$ if the sample contains the spatial frequency vector $\mathbf{K}$. The scattered anti-Stokes wave is collected by the microscope objective if $k_{\|, a S} \leq k_{a S}$ NA. c) Classical phase-matching condition for a homogenous sample, i.e. $\mathbf{K}=\mathbf{0}$ under plane-wave excitation. d) Conventional representation of the transfer function displaying the $k_{z}$-projection of the Ewald's spheres of sub-Figs. a) and b).

in a $4 f$ configuration (see Fig. 2). Hereafter, we denote by object space the semi-infinite medium before the objective and by image space the semi-infinite medium after the tube lens. The optical axis $z$ is oriented from the object to the image space and we note $\mathbf{r}_{\|}=(x, y)$ the transverse coordinates. For convenience, the phase origin in the object space is taken at the intersection of the optical axis and the object focal plane while the phase origin in the image plane is taken at the intersection of the optical axis and the image focal plane. Last, we assume that the background medium of the image space is the same as that of the object space.

\subsection{Modeling the CARS radiation in the object space}

To model the anti-stokes field recorded at the image plane of the microscope, one needs to evaluate the radiated field at $\mathbf{R}$ in the object space comprised between the sample and the microscope objective $[12,31,32]$. As shown in the previous section, the CARS radiation satisfies Eq. (5). The observation point being now a few wavelength away $\left(>\lambda_{a S}\right)$ from the sample it is appropriate to use the angular spectrum representation of the Green tensor [27,31,33], restricted to the plane waves propagating in the forward direction,

$$
\overleftrightarrow{\mathbf{G}}(\mathbf{R}, \mathbf{r}) \approx \frac{i}{8 \pi^{2}} \int \mathrm{d} \mathbf{k}_{\|} h\left(\mathbf{k}_{\|}\right) \overleftrightarrow{\mathbf{M}}\left(\mathbf{k}_{a S}\right) \exp \left[i \mathbf{k}_{a S} \cdot(\mathbf{R}-\mathbf{r})\right]
$$

where $\mathbf{k}_{a S}=\mathbf{k}_{\|}+\gamma \mathbf{z}$ with $\gamma=\sqrt{k_{\mathrm{as}}^{2}-k_{\|}^{2}}$ and $h$ equal to $1 / \gamma$ if $k_{\|}<k_{\mathrm{aS}}$ and 0 elsewhere.

Introducing Eq. (16) into Eq. (5) yields, after some reordering,

$$
\mathbf{E}_{a S}(\mathbf{R})=\frac{i k_{a S}^{2}}{8 \pi^{2}} \int \mathrm{d} \mathbf{k}_{\|} h\left(\mathbf{k}_{\|}\right) \overleftrightarrow{\mathbf{M}}\left(\mathbf{k}_{a S}\right) \exp \left(i \mathbf{k}_{a S} \cdot \mathbf{R}\right) \int \mathrm{d} \mathbf{r} \exp \left(-i \mathbf{k}_{a S} \cdot \mathbf{r}\right) \mathbf{P}_{a S}^{(3)}(\mathbf{r})
$$

Then, the right-hand side of Eq. (17) can be rewritten as a sum of plane waves whose amplitude 


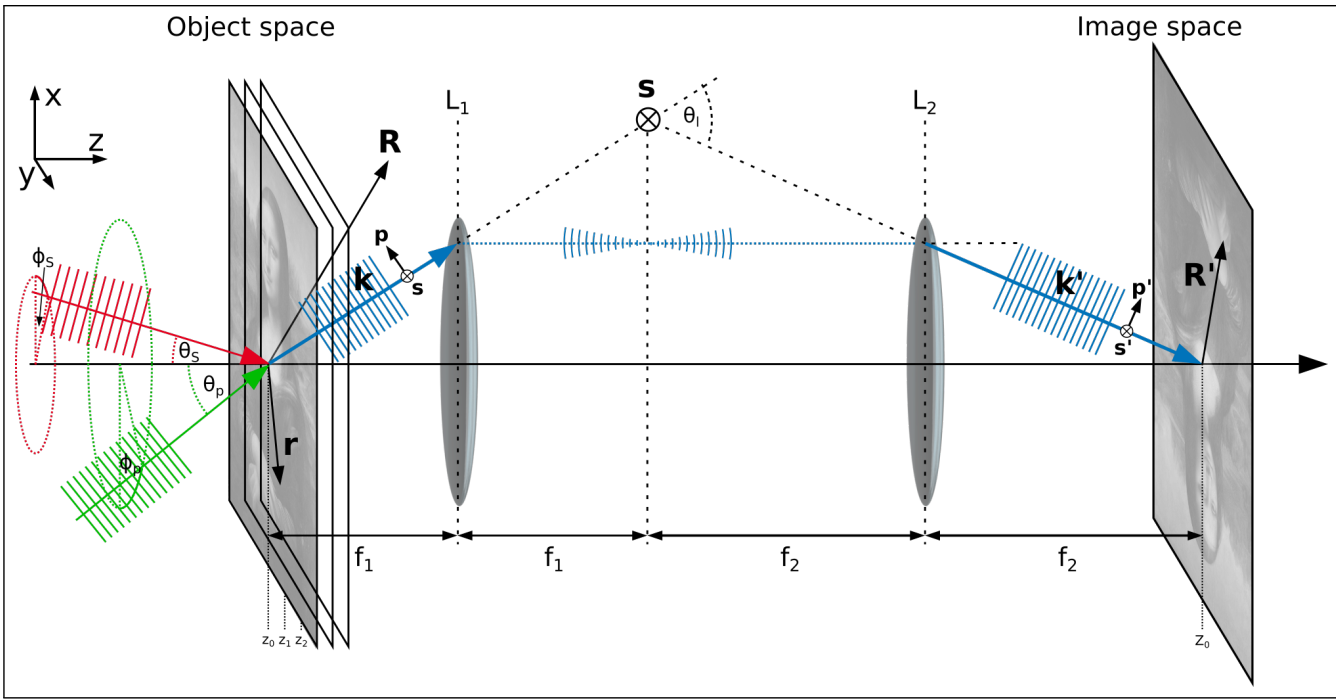

Fig. 2. Declaration of variables: $\mathbf{r}$ coordinates of the object; $\mathbf{R}$ coordinates of the far-field; $\mathbf{R}^{\prime}$ coordinates of the image plane; $\mathbf{k}$ wavevector in the object space; $\mathbf{k}$ ' wavevector in the image space; $\mathbf{p}^{\left({ }^{\prime}\right)}, \mathbf{s}^{\left({ }^{\prime}\right)}$ unit vectors of plane wave polarization; $\theta_{S}$ polar angle of the Stokes beam; $\theta_{p}$ polar angle of the pump beam; $\phi_{S}$ azimuth angle of the Stokes beam; $\phi_{p}$ azimuth angle of the pump beam; $\theta_{l}$ polar angle alteration after both lenses; $f_{1}$ focal length of lens $\mathrm{L}_{1}$; $f_{2}$ focal length of lens $\mathrm{L}_{2} ; z_{0}, z_{1}, z_{2}$ axial sample coordinates; $Z_{0}$ axial image coordinates.

are related to the 3D Fourier transform of the anti-Stokes polarization,

$$
\mathbf{E}_{a S}(\mathbf{R})=i \pi k_{a S}^{2} \int \mathrm{d} \mathbf{k}_{\|} \overleftrightarrow{\mathbf{M}}\left(\mathbf{k}_{a S}\right) e^{i \mathbf{k}_{a S} \cdot \mathbf{R}} h\left(\mathbf{k}_{\|}\right) \tilde{\mathbf{P}}_{a S}^{(3)}\left(\mathbf{k}_{a S}\right)
$$

\subsection{Modeling the CARS radiation at the image plane}

We now study the field in the image space of the microscope. The plane waves forming the field in the object space are modified by the imaging system because of the magnification $M$ brought by the objective and tube lens, $M=-f_{2} / f_{1}[31]$.

1. First, the wavevectors of the plane waves forming the field in the object space are changed from $\mathbf{k}_{a S}$ to $\mathbf{k}_{a S}^{\prime}=\mathbf{k}_{\|} / M+\gamma^{\prime} \mathbf{z}$ with $\gamma^{\prime}=\sqrt{k_{a S}^{2}-k_{\|}^{2} / M^{2}}$.

2. Second, the vectorial amplitude of the plane wave is rotated to remain normal to the novel direction of propagation $\mathbf{k}_{a S}^{\prime}$ and dimmed to ensure energy conservation. Introducing the $(\mathbf{s}, \mathbf{p})$ basis related to $\mathbf{k}_{a S}, \mathbf{s}=\mathbf{z} \times \mathbf{k}_{a S} / k_{a S}$ and $\mathbf{p}=\mathbf{s} \times \mathbf{k}_{a S} / k_{a S}$, when the plane wave direction $\mathbf{k}_{a S}$ is changed into $\mathbf{k}_{a S}^{\prime}$, the field amplitude $\mathbf{E}=E^{s} \mathbf{s}+E^{p} \mathbf{p}$ is transformed into $\mathbf{E}^{\prime}=M^{-1} \sqrt{\gamma / \gamma^{\prime}}\left(E^{s} \mathbf{s}+E^{p} \mathbf{p}^{\prime}\right)$ where $\mathbf{p}^{\prime}=\mathbf{s} \times \mathbf{k}_{a S}^{\prime} / k_{a S}$ and the coefficient $M^{-1} \sqrt{\gamma / \gamma^{\prime}}$ ensures the energy conservation. To perform this rotation we introduce the tensor, $\overleftrightarrow{\mathbf{Q}}\left(\mathbf{k}_{a S}^{\prime}, \mathbf{k}_{a S}\right)$ which rotates vectors by an angle $\theta_{l}$ defined as $\sin \theta_{l}=\left|\mathbf{k}_{a S} \times \mathbf{k}_{a S}^{\prime}\right| / k_{a S}^{2}$ about the axis $(\mathbf{k} \times \mathbf{z}) /|\mathbf{k} \times \mathbf{z}|$.

3. Third, some plane waves of the field in the object space are blocked by the diaphragm at the pupil plane. $h$ is now replaced by $h_{\mathrm{NA}}$ which verifies $h_{\mathrm{NA}}\left(\mathbf{k}_{\|}\right)=1 / \gamma$ for $k_{\|}<k_{a S} \mathrm{NA}$ and 0 elsewhere. 
4. Fourth, our imaging system is aplanatic (the objective satisfies the Sine-Abbe condition). In other word, the lenses have been optimized so that plane waves that interfere constructively at a point of the object focal plane will interfere constructively at a point of the image focal plane (See appendix A for the transition $\mathbf{R} \rightarrow \mathbf{R}^{\prime}$ ).

Under these four conditions, the field at $\mathbf{R}^{\prime}$ in the image plane, stemming from Eq. (18) becomes a sum of plane waves with wavevector $\mathbf{k}_{a S}^{\prime}$ and amplitude proportional to $\tilde{\mathbf{P}}_{a S}^{(3)}\left(\mathbf{k}_{a S}\right)$,

$$
\mathbf{E}_{a S}\left(\mathbf{R}^{\prime}\right)=i \pi \frac{k_{a S}^{2}}{M} \int \mathrm{d} \mathbf{k}_{\|} e^{i \mathbf{k}_{a S}^{\prime} \cdot \mathbf{R}^{\prime}} \sqrt{\frac{\gamma}{\gamma^{\prime}}} h_{\mathrm{NA}}\left(\mathbf{k}_{\|}\right) \overleftrightarrow{\mathbf{Q}}\left(\mathbf{k}_{a S}^{\prime}, \mathbf{k}_{a S}\right) \overleftrightarrow{\mathbf{M}}\left(\mathbf{k}_{a S}\right) \tilde{\mathbf{P}}_{a S}^{(3)}\left(\mathbf{k}_{a S}\right)
$$

At the image plane, $\mathbf{R}^{\prime}=\left(M \mathbf{R}_{\|}, 0\right)$, one can write Eq. (19) in a way that facilitates its interpretation [27]. Introducing the three dimensional pupil function,

$$
\mathcal{P}(\mathbf{k})=\mathcal{H}\left(\mathbf{k}_{\|}\right) \delta\left[k_{z}-\gamma\left(\mathbf{k}_{\|}\right)\right] \text {where } \mathcal{H}\left(\mathbf{k}_{\|}\right)=\frac{k_{a S}^{2}}{M} \sqrt{\frac{\gamma}{\gamma^{\prime}}} h_{\mathrm{NA}}\left(\mathbf{k}_{\|}\right)
$$

with $\mathbf{k}=\mathbf{k}_{\|}+k_{z} \mathbf{z}$, Eq. (19) reads,

$$
\mathbf{E}_{a S}\left(M \mathbf{R}_{\|}\right)=i \pi \mathcal{F}_{3 D}^{-1}\left[\mathcal{P}(\mathbf{k}) \overleftrightarrow{\mathbf{Q}}\left(\mathbf{k}^{\prime}, \mathbf{k}\right) \overleftrightarrow{\mathbf{M}}(\mathbf{k}) \tilde{\mathbf{P}}_{a S}^{(3)}(\mathbf{k})\right]\left(\mathbf{R}_{\|}\right)
$$

Recalling Eq. (13) in Eq. (21), we observe that, the anti-Stokes field at the image plane of the microscope permits to recover the sample density spectrum $\tilde{N}$ on a cap of sphere (of radius $k_{a S}$ with transverse projection the disk of radius $k_{a S} \mathrm{NA}$ ) translated by $2 \mathbf{k}_{p}-\mathbf{k}_{S}$. We retrieve the Fourier analysis provided in the first section, Fig. 1.

Equation (19) is convenient for linking the image field to the three-dimensional sample. Yet, to simulate the field at the image plane on the regular square meshing of the camera pixels, it is more interesting to rewrite the expression so as to use two-dimensional fast Fourier transforms [34].

$$
\mathbf{E}_{a S}\left(M \mathbf{R}_{\|}\right)=\frac{i}{2} \int \mathrm{d} z \mathcal{F}_{2 D}^{-1}\left[\mathcal{H}\left(\mathbf{k}_{\|}\right) e^{-i \gamma z} \overleftrightarrow{\mathbf{Q}}\left(\mathbf{k}_{a S}^{\prime}, \mathbf{k}_{a S}\right) \overleftrightarrow{\mathbf{M}}\left(\mathbf{k}_{a S}\right) \mathcal{F}_{2 D}\left[\mathbf{P}_{a S}^{(3)}\left(\mathbf{r}_{\|}, z\right)\right]\right]
$$

where we have used that $\tilde{\mathbf{P}}_{a S}^{(3)}(\mathbf{k})=\frac{1}{2 \pi} \int e^{-i \gamma z} \mathcal{F}_{2 D}\left[\mathbf{P}_{a S}^{(3)}\left(\mathbf{r}_{\|}, z\right)\right]\left(\mathbf{k}_{\|}\right) \mathrm{d} \mathbf{k}_{z}$. With the definition of the 2D Fourier transform:

$$
\mathcal{F}_{2 D}(f)=\frac{1}{4 \pi^{2}} \int f\left(\mathbf{r}_{\|}\right) e^{-i \mathbf{k}_{\|} \cdot \mathbf{r}_{\|}} \mathrm{d} \mathbf{r}_{\|} \text {and } \mathcal{F}_{2 D}^{-1}(g)=\int g\left(\mathbf{k}_{\|}\right) e^{i \mathbf{k}_{\|} \cdot \mathbf{r}_{\|}} \mathrm{d} \mathbf{k}_{\|}
$$

\section{Inversion procedure for CARS Fourier ptychography microscopy (CARS FPM)}

The link between the field at the image plane and the sample being established, we now consider an imaging approach where the scatterer density is reconstructed from several CARS images obtained under $L$ different excitations with various pump and Stokes wavevectors. If the complex field $\mathbf{E}_{a S}$ is recorded at the image plane (using an interferometric set-up similar to that used in tomographic diffraction microscopy [30,35] for example), a direct linear reconstruction of the sample complex density can be developed easily using Eqs. (21) and (13) as in Tomographic Diffraction Microscopy [30].

On the other hand, if only the intensity $\left|\mathbf{E}_{a S}\right|^{2}$ is recorded at the image plane, the reconstruction issue resembles better that of Optical Fourier Ptychography Microscopy (FPM) where the complex index of refraction is retrieved numerically from several intensity images of the sample obtained under various angles of illumination in a transmission microscope [25,36-41].

Unfortunately, the direct adaptation of the FPM inversion algorithms to CARS FPM is impossible for one major reason. In transmission FPM, the scattered field carrying the sample 
information interferes with the specularly transmitted beam. This interference brings important clues on the scattered field phase. In CARS FPM on the contrary, there is no reference field at the anti-Stokes wavelength with which the emitted CARS field could interfere (in this work we neglect the possible non-resonant radiation of the glass slide holder and assume that the sample is floating in air). We have thus derived an original intensity-based reconstruction algorithm, inspired from [42].

The inversion scheme is based on Eq. (22). To simplify its presentation, we restrict here the sample to a thin slice of material at $z=z_{0}$. The unknown third order susceptibility is thus written as, $\overleftrightarrow{\chi^{(3)}}(\mathbf{r}) \approx N\left(\mathbf{r}_{\|}\right) \delta\left(z-z_{0}\right) \overleftrightarrow{\chi^{(3)} \prime}$ where $\overleftrightarrow{\chi^{(3)} \prime}$ is known. The inversion scheme aims at reconstructing the scatterer density $N\left(\vec{r}_{\|}\right)$at the plane $z=z_{0}$.

The scatterer density is estimated iteratively so as to minimize the distance between the recorded image data $I_{l}^{\text {mes }}$ for the $l=1, \cdots, L$ illuminations conditions (pump or Stokes varying incident angle) and the simulated intensity at the camera plane,

$$
F(N)=\left.\left.W^{-1} \sum_{l=1}^{L} \int \mathrm{d}\left(M \mathbf{R}_{\|}\right)\left|I_{l}^{\mathrm{mes}}-\right| \mathbf{E}_{a S, l}[N]\right|^{2}\right|^{2}
$$

where $W=\sum_{l} \int \mathrm{d}\left(M \mathbf{R}_{\|}\right)\left|I_{l}^{\text {mes }}\right|^{2}$ and the dependence of $I_{l}^{\text {mes }}$ and $\mathbf{E}_{a S, l}$ with respect to $M \mathbf{R}_{\|}$and $N$ will be made implicit. A conjugate gradient method is used to minimize the cost functional, Eq. (24). At iteration $n, N$ can be updated using the formula,

$$
N^{n}\left(\mathbf{r}_{\|}\right)=N^{n-1}\left(\mathbf{r}_{\|}\right)+\alpha_{\text {eff }} d^{n}\left(\mathbf{r}_{\|}\right)
$$

where $\alpha_{e f f}$ is a real derived in appendix D and $d_{n}$ is the Polak-Ribière descent [43] provided by,

$$
d^{n}\left(\mathbf{r}_{\|}\right)=g^{n}\left(\mathbf{r}_{\|}\right)+\frac{\int \mathrm{d} \mathbf{r}_{\|} g^{n}\left(g^{n}-g^{n-1}\right)^{*}}{\int \mathrm{d} \mathbf{r}_{\|} g^{n-1}\left(g^{n-1}\right)^{*}} d^{n-1}
$$

The gradient at iteration $n, g^{n}$, is provided by (see appendix $\mathrm{C}$ for its derivation)

$$
g^{n}\left(\mathbf{r}_{\|}, z_{0}\right)=\frac{4 i}{W} \sum_{l} \mathbf{u}_{l}^{*}\left(\mathbf{r}_{0}\right) \cdot \mathcal{F}_{2 D}^{1}\left\{e^{i \gamma z_{0}} \mathcal{H}\left(\mathbf{k}_{\|}\right) \overleftrightarrow{\mathbf{Q}}\left(\mathbf{k}_{a S}, \mathbf{k}_{a S}^{\prime}\right) \overleftrightarrow{\mathbf{M}}\left(\mathbf{k}_{a S}^{\prime}\right) \mathcal{F}_{2 D}\left[t_{l} \mathbf{E}_{a S, l}\right]\right\}\left(\mathbf{r}_{\|}\right)
$$

where $t_{l}^{n-1}=I_{l}^{\text {mes }}-\left|E_{a S, l}^{n-1}\right|^{2}$ is the residue at the $(n-1)^{t h}$ iteration. The inversion scheme is easily extended to samples that are made of $J$ slices along the $z$ axis by introducing $\left\{N_{1} \ldots N_{J}\right\}$ the unknown densities at each slice. At iteration $n, N_{j}$ is modified using Eqs. (25) and (27) where we recall that the field at the image plane $\mathbf{E}_{a S, l}^{n-1}$ is calculated with Eq. (22) with the estimations of the $J$ scatterer densities $\left\{N_{j}\right\}$ obtained at the previous iteration.

\section{Synthetic CARS Fourier ptychography}

To test the reconstruction scheme, we consider the CARS FPM experiment depicted in Fig. 5 in which the Stokes and Pump angles defined by $\mathbf{k}_{p, S}=k_{p, S,[}\left[\sin \theta_{p, S}, \cos \phi_{p, S} \mathbf{x}+\sin \theta_{p, S} \sin \phi_{p, S} \mathbf{y}+\right.$ $\left.\cos \theta_{p, S} \mathbf{z}\right]$ can be modified. To this aim, we consider two beams at $\lambda_{S}=1030 \mathrm{~nm}$ and $\lambda_{p}=796 \mathrm{~nm}$ whose incidence angles are controlled by laser-scanning mirrors in combination with a $4 f$-lens system [2]. Before the mirrors, the polarization of the beams is fixed along the $x$ direction. After the mirrors, the excitation fields at the sample become [2],

$$
\mathbf{E}_{p, S}\left(\mathbf{r}, \phi_{p, S}, \theta_{p, S}\right)=E_{0} \sqrt{n_{p, S} \cos \theta_{p, S}}\left[\begin{array}{c}
\cos ^{2} \phi_{p, S} \cos \theta_{p, S}+\sin ^{2} \phi_{p, S} \\
-\left(1-\cos \theta_{p, S}\right) \sin \phi_{p, S} \cos \phi_{p, S} \\
-\sin \theta_{p, S} \cos \phi_{p, S}
\end{array}\right] \exp \left[i \mathbf{k}_{p, S} \cdot \mathbf{r}\right] .
$$


The CARS radiation at $\lambda_{a S}=649 \mathrm{~nm}$ corresponding to a Raman shift of $2850 \mathrm{~cm}^{-1}$ is collected by a microscope objective of numerical aperture NA $=0.2$ and magnification $M=10$ after a polarizer which detects only the $x$ component of the field. Note that, the observation angles being small, the $\mathrm{x}$-component of the CARS field depends essentially on the $\mathrm{x}$-component of the polarization $\mathbf{P}^{(3)}$. We consider two different scanning schemes, the pump (Stokes) beam is fixed and the Stokes (pump) beam is varied. In all cases, the fixed beam is $x$-polarized and directed along the optical axis. In the Stokes scanning configuration, because of the properties of the third order susceptibility tensor $\overleftrightarrow{\chi^{(3)} \text { ' }}$ the $x$-component of the polarization will depend only the first coefficient $\chi_{1111}^{(3)}$ as shown in appendix B. On the contrary, in the pump scanning configuration, it will depend on $\chi_{1111}^{(3)}, \chi_{1221}^{(3)}$ and $\chi_{1331}^{(3)}$. For computation we set $\chi_{1111}^{(3)}=1$ while $\chi_{1221}^{(3)}=1 / 3$ and $\chi_{1331}^{(3)}=1 / 3$.

The CARS images are simulated on $201 \times 201$ pixels with stepsize $500 \mathrm{~nm}$ at the image plane using Eqs. (25)-(28) and deteriorated with Poisson noise assuming a maximum mean value of 1000 photons for the brightest pixel (which corresponds to a noise standard deviation of $3.2 \%$ ).

The sample is a $2 \mathrm{D}$ spoke-pattern placed at $z_{0}=20 \mu \mathrm{m}$. This kind of sample is particularly convenient for investigating the resolution in the transverse plane [44,45], see Fig. 4(a) for the real and imaginary (Fig. 4(e)) parts of the ground truth image.

All reconstructions are launched with an initial estimate, $N^{0}=0.01+i 0.01$ and the iterations are stopped after 400 iterations. The matlab inversion algorithm was run on an Intel(R) Xeon(R) CPU E5-1620 v4 @ 3.50 GHz. The computation time is estimated to $8 \mathrm{~min}, 18 \mathrm{~min}, 58 \mathrm{~min}$ for 9, 49, 169 images, respectively. A video (Visualization 1) is provided within the supplemental material showing the development of the real and imaginary part of the scatterer distribution after each iteration. The matlab script for the computation of wide-field CARS images and the sample reconstruction will be provided on demand - please contact the corresponding authors.

In a first experiment, the Stokes angles are varied so that $\mathbf{k}{ }_{\| S}$ points on a regular square grid with step $0.06 k_{a S}$ while the pump angle is kept at $\theta_{p}=0^{\circ}$. Figure 3 shows several synthetic images obtained for different illuminations. Introducing Eq. (13) in Eq. (19) shows that, for each illumination, the CARS image yields information on a specific portion of $\tilde{N}$. In the bottom plot, we display $|\tilde{N}|$ and indicate with blue circles the Fourier domain that is scanned when varying the Stokes angle according to Eq. (19) and Eq. (13). The disk of radius $k_{a s}$ NA corresponds to the region covered when varying the observation wavevector $\mathbf{k}_{\|}$while its center is given by the transverse component of $2 \mathbf{k}_{p}-\mathbf{k}_{S}$ which simplifies to $-\mathbf{k}_{\| S}$ in the Stokes scanned configuration. The overlapping between these disks is about $75 \%$ as required in ptychography [41].

In the first experiment, only 9 images at low Stokes angles, NA $=0.26$, feed the inversion scheme. The reconstruction displayed in Figs. 4(b) and 4(f) shows that both the real and imaginary part of $N$ are recovered. In Figs. 4(c) and 4(g), 49 images are taken in the inversion scheme with increased Stokes angles, NA $=0.4$, and yield better resolved reconstruction. Last, we improve further the resolution, by considering the pump scanning configuration, Figs. 4(d) and 4(h). In this configuration, the maximum angular excursion of the pump beam is taken similar to that of the Stokes scanning experiment. Yet, each disk in the Fourier plane being translated by $2 \mathbf{k}_{\| p}$, the accessible Fourier domain in the pump scanning technique is much larger than that of the Stokes configuration and corresponds to $\mathrm{NA}=0.7$. Note that to ensure the same overlapping, $13 \times 13$ images were taken in this case.

The sensitivity to noise is studied in Fig. 4 where the residue of the cost functional $F$ is plotted at the end of Fig. 4 for images with $3.2 \%, 10 \%$ and $31.6 \%$ shot noise levels. Unsurprisingly, the larger the number of images taken in the reconstruction, the better the robustness to noise. Once the complex density has been retrieved, the amplitude and phase of the wide-field CARS images at the camera plane can be simulated, as seen in Figs. 4(i) and 4(j). Thus, reference-less phase-retrieval comes as a natural byproduct of CARS FPM. This property is important as the 

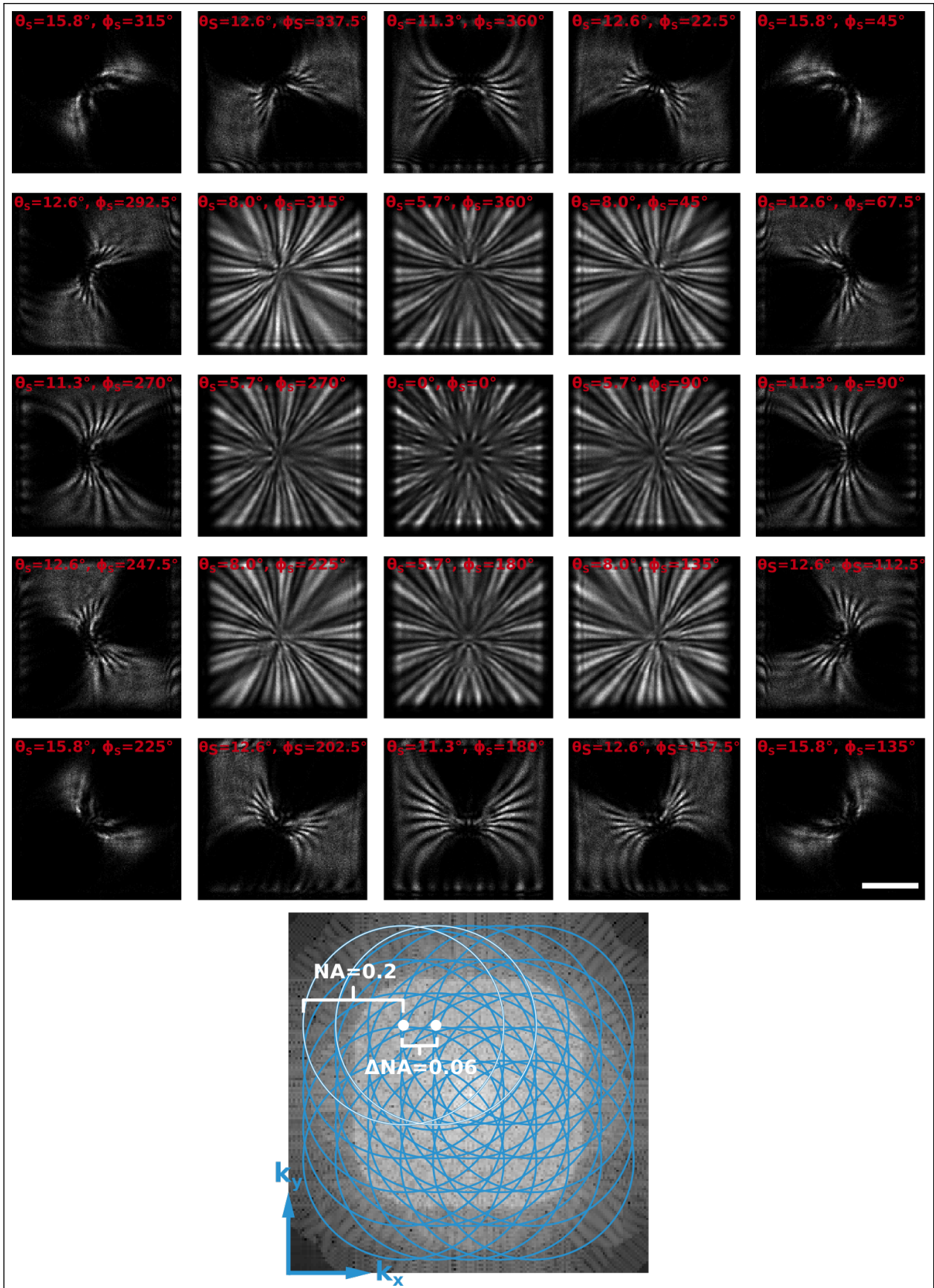

Fig. 3. Simulated 25 wide-field CARS images for different illumination angles of the Stokes beam and normal pump beam incidence. The images were calculated using Eq. (22). Parameters - Raman shift: $2850 \mathrm{~cm}^{-1}, \lambda_{S}=1030 \mathrm{~nm}, \lambda_{p}=796 \mathrm{~nm} \lambda_{a S}=649 \mathrm{~nm}$, magnification: 10x, defocus: $z_{0}=20 \mu \mathrm{m}$, detection NA: 0.2 . While normal incidence of the pump beam is assumed the illumination angles of the Stokes beam are outlined within each image ( $\theta_{S}$ polar angle, $\phi_{S}$ azimuthal angle). The imaginary and real part of the ground truth (GT) of the 2D sample is displayed in Fig. 4 a and e. Poisson noise was added assuming that the brightest pixel on the camera collects a mean value of 1000 photons. The scale bar equals $200 \mu \mathrm{m}$. The blue circles on top of the Fourier images of the anti-Stokes polarization is intended as a guide to the eye to estimate the overlap of neighboring illuminations. 

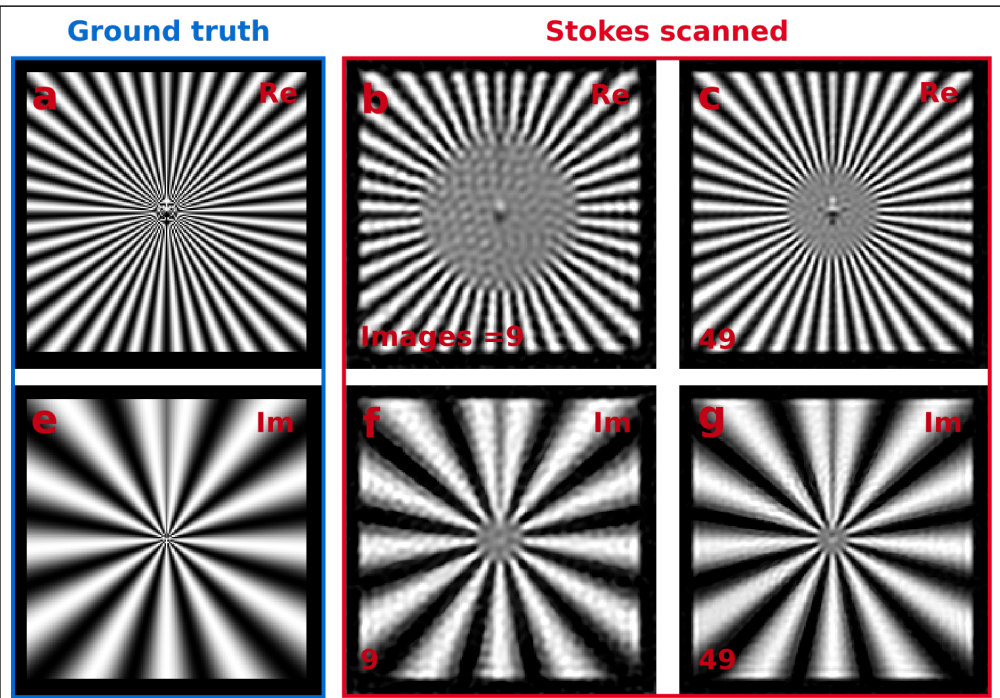

pump scanned
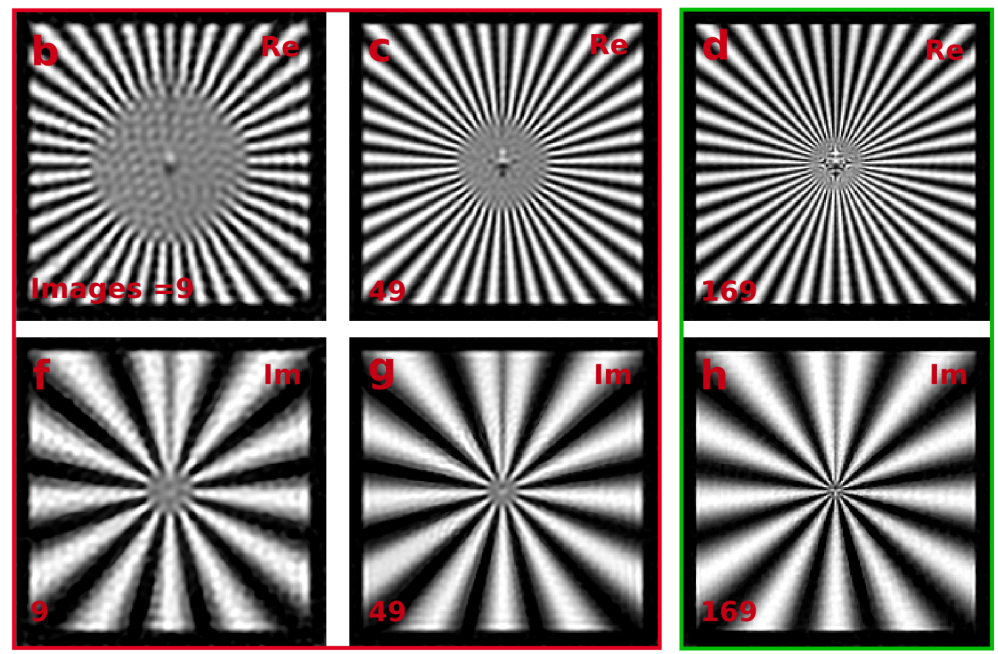

Amplitude
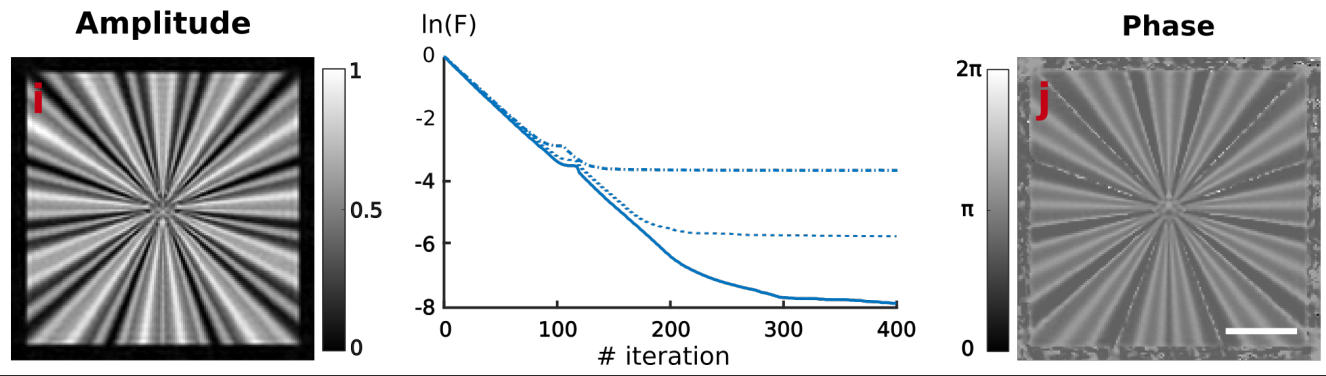

Fig. 4. Reconstruction results: The real and imaginary part of the sample's ground truth (GT) are displayed in the sub-Figs. a and e, respectively. The images $b$ and $f$ were reconstructed from the center 9 wide-field CARS images presented in Fig. 3. Sub-Figs. c and g display the corresponding reconstruction results for 49 wide-field CARS images. Scanning the pump beam the images $d$ and $h$ were obtained. Note that though more images were included in the reconstruction the maximum excitation angle $\theta_{p}$ equals the maximum excitation angle in Stokes scanned configuration in sub-Figs. $\mathrm{c}$ and g. The added Poisson noise assumes 1000 photons for the brightest image pixel, i.e. a standard deviation of $3.2 \%$. Sub-Figs. i and j display the calculated in-focus amplitude and phase images resulting from the reconstructed complex sample structure of sub-Figs. $\mathrm{c}$ and $\mathrm{g}$. The natural logarithm of the residue of the cost functional $F$ as defined in Eq. (24) is plotted in the lower center of the Fig. as a function of the iteration number for 49 Stokes scanned wide-field CARS images. The continuous, dotted and dash-dotted lines account for a standard deviation of $3.2 \%, 10 \%$ and $31.6 \%$ of added Poisson noise, respectively. The blue and white scale bar equal $10 \mu \mathrm{m}$ and $100 \mu \mathrm{m}$, respectively. 
imaginary part of the third order susceptibility is proportional to the Raman polarization and follows linearly the scatterer concentration [46].

This simulated experiment shows that CARS FPM is able to recover the complex nature of $N$ with improved resolution, even though it does not benefit from the interference with a reference field as in linear FPM. However, CARS FPM is not always s uccessful. An important condition is that the images should vary significantly with the Stokes (or pump) illumination angles. In the Visualization 1 (supplementary information), one observes that the reconstruction starts at points where the intensity variation with changing illumination angle is the most pronounced. When the sample is made of large homogeneous regions or, conversely, of sparse in-focus single emitters, the images do not depend on the incident angles and we have observed (not shown) the reconstruction algorithm fails. The variation of the intensity with respect to the angles of illumination is essentially due to the interference phenomenon between the fields radiated by the scatterers. A gentle defocusing and low detection NA may benefit to the reconstruction scheme by increasing the domain over which the fields i nterfere. In addition, the intensity variations should be different for the real and imaginary scatterer density $N=N^{\prime}+i N^{\prime \prime}$ for the inversion scheme to distinguish the two contributions. Obviously, if $N^{\prime}(\mathbf{r})=a N^{\prime \prime}(\mathbf{r})$, CARS FPM will not be able to extract the complex value of $N$.

Although not universal, CARS FPM is expected to be particularly efficient for imaging thin tissue sections as in [9] addressing CH-stretching vibrations. Obviously, these samples do not suffer from sparsity. Further, the nuclei, cytoplasm and the cell walls are made of different densities of $\mathrm{CH}_{2}$ and $\mathrm{CH}_{3}$ groups which are currently used to generate virtual $\mathrm{H} \& \mathrm{E}$ images [47]. Apart from the ever present nonresonant background, the methylene and methyl groups display different amount of real and imaginary contributions for a given Raman shift so that a sufficient spatially varying distribution of real and imaginary scatterer density should be provided.

We finish this theoretical and numerical study with some considerations on the feasibility of this widefield technique and an estimation of the required laser parameters.

\section{Designing the implementation of CARS FPM, study of the field of view}

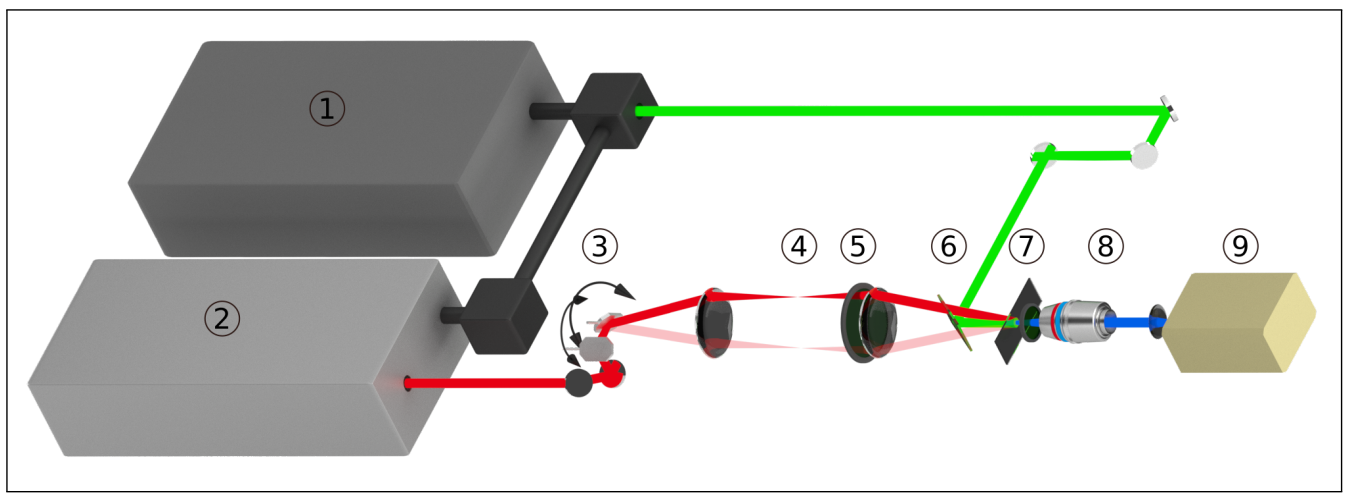

Fig. 5. Proposed experimental implementation:1 High power pump laser at 1029-1065 nm (FWHM $<2 \mathrm{~nm}$ ) with a repetition rate $10 \mathrm{kHz}-1 \mathrm{MHz}$ and a pulselength $1 \mathrm{ps}, 2$ Optical parametric amplifier (OPA) tunable within $730-1010 \mathrm{~nm}\left(200-4000 \mathrm{~cm}^{-1}\right), 3$ laser-scanning mirrors, $44 f$-imaging system, 5 linear polarizer, 6 dichroic beam combiner, 7 sample and linear polarizer as well as dielectric filters, 8 low NA $4 f$-system, 9 low noise (cooled) CCD camera.

An outline for a possible experimental configuration of CARS FPM is displayed in Fig. 5. Here, a dual-color laser system is employed for excitation of wide-field CARS radiation. The 
radiation of the Stokes (or pump) beam is angle-scanned at the position of the sample using a combination of laser scanning mirrors and a $4 f$-imaging system, e.g. composed of 2 lenses. Alternatively, the Stokes beam could be scanned with laser scanning mirrors and an array of mirrors as demonstrated for linear FPM [48] to avoid aberrations introduced by the lenses.

The pump (or Stokes) beam is adapted in size, e.g. by means of a telescope, and superimposed with the Stokes beam in time and space using a delay stage. A dielectric and a linear polarization filter is positioned just after the sample to collect only x-polarized radiated CARS light and to prevent the radiation of the excitation laser from entering the collection objective lens with potential multi-color generation while interacting with the glass, e.g. by means of four-wavemixing or auto-fluorescence. For collecting the anti-Stokes radiation a low NA objective lens is used in combination with a second lens to form an image at a low noise CCD camera.

The major crux of the implementation lays in the definition of the parameters of the lasers for a given field of view (FOV). The peak power and repetition rate have to be optimized to activate the third-order nonlinear excitation on the FOV while minimizing sample damage mechanism as heating or multi-photon-absorption. As a reference, we consider the widefield experiment conducted in [21]. Using a laser with repetition rate $\rho=76 \mathrm{MHz}$, pulse full width at half maximum $\tau=3 \mathrm{ps}, \lambda_{a s}: 600 \mathrm{~nm}\left(2850 \mathrm{~cm}^{-1}\right)$, average power $P_{p,(S)}=150 \mathrm{~mW}$, the authors were able to image test samples up to 33 frames-per-second (fps) and biological samples up to 2 fps with a FOV $S=20 \times 20 \mu \mathrm{m}^{2}$. Now, the CARS signal per unit area (SPUA) is linked to these parameters as,

$$
\mathrm{SPUA}=\frac{\eta \rho}{\lambda_{\mathrm{aS}}^{4}} \frac{\mathrm{P}_{\mathrm{p}}^{2} \mathrm{P}_{\mathrm{S}}}{(\mathrm{S} \rho \tau)^{3}},
$$

the coefficient $\eta$ depending on the image integration time, the quantum yield and the collection objective. We propose to upgrade this experiment in order to image a larger FOV. We consider a realistic Yb-fiber laser system with an optical parametric amplifier (OPA) meeting the key parameters: $\rho=10 \mathrm{kHz}, \tau=3 \mathrm{ps}, \lambda_{a S}: 650 \mathrm{~nm}\left(2850 \mathrm{~cm}^{-1}\right), P_{p,(S)}=1 \mathrm{~W}$. To compute the expected FOV for the second system these parameters are inserted into Eq. (29) assuming the same SPUA and $\eta$ as the reference experiment. With such a $\mathrm{kHz}$ ps laser system, a FOV of about $1 \mathrm{~mm}^{2}$ could possibly be achieved with the same frame rates and image quality as in the reference experiment [21].

\section{Conclusion}

In conclusion, we proposed and derived a novel CARS wide field imaging scheme referred as CARS Fourier ptychography (CARS FPM). By scanning the incident angles of the incoming Stokes or pump beams, we show that it is possible to retrieve a wide field chemical image featuring an improved resolution as compared to a single illumination as previously reported in the literature. For this we have developed a full vectorial model of the wide field CARS image of a microscope in a $4 f$ configuration together with a novel inversion procedure able to reconstruct the sample density from several CARS images recorded for various excitations angles. We have shown that CARS FPM allows the reconstruction of both real and imaginary parts of the complex third order susceptibility, the latter being linear with the chemical concentration and giving directly access to the Raman information. We have finally proposed a realistic experimental scheme that would be suitable to perform CARS FPM. Possible extensions of this work could include a 3D sample reconstruction that is possible using a modified inversion algorithm and the reconstruction of the pupil function [40] allowing aberration-free wide field CARS images.

\section{Appendix A - phase acquisition of planes waves passing a $4 f$ system}

The phase acquired by the plane wave propagating from $\mathbf{r}$ to $\mathbf{R}^{\prime}$ (see Fig. 6) in paraxial approximation is provided by : 


$$
\begin{aligned}
\phi_{4 f} & =\mathbf{k}\left(\mathbf{r}_{1}-\mathbf{r}\right)+\mathbf{k}\left(\mathbf{u}-\mathbf{r}_{1}\right)+\mathbf{k}^{\prime}\left(\mathbf{R}_{1}-\mathbf{u}\right)+\mathbf{k}^{\prime}\left(\mathbf{R}^{\prime}-\mathbf{R}_{1}\right) \\
& =\mathbf{k}^{\prime} \mathbf{R}^{\prime}-\mathbf{k r}+\mathbf{k u}-\mathbf{k}^{\prime} \mathbf{u} \\
& =\mathbf{k}^{\prime} \mathbf{R}^{\prime}-\mathbf{k r}+\underbrace{2 f_{1} k_{0}+2 f_{2} k_{0}}_{\text {const } \forall \mathbf{k}}
\end{aligned}
$$

where it was used that

$$
\mathbf{k} \approx\left[\begin{array}{c}
\mathbf{k}_{\|} \\
k_{0}\left(1-\frac{\mathbf{k}_{\|}^{2}}{2 k_{0}}\right)
\end{array}\right] \quad \mathbf{k}^{\prime} \approx\left[\begin{array}{c}
\mathbf{k}_{\|}^{\prime} \\
k_{0}\left(1-\frac{\mathbf{k}_{\|}^{\prime 2}}{2 k_{0}}\right)
\end{array}\right] \quad \mathbf{u}=\left[\begin{array}{c}
\frac{f_{1} \mathbf{k}_{\|}}{k_{0}} \\
2 f_{1}
\end{array}\right]=\left[\begin{array}{c}
\frac{f_{2} \mathbf{k}_{\|}^{\prime}}{k_{0}} \\
2 f_{2}
\end{array}\right]
$$

Thus, the phase relation between any point $\mathbf{r}_{1}$ and $\mathbf{R}^{\prime}$ is provided by a constant plus $\mathbf{k}^{\prime} \mathbf{R}^{\prime}-\mathbf{k r}$. If the constant term is neglected Eq. (30) results the missing link for the transition from the far field coordinate $\mathbf{R}$ to the image space coordinate $\mathbf{R}^{\prime}$ (see Eqs. (18) and (19)).

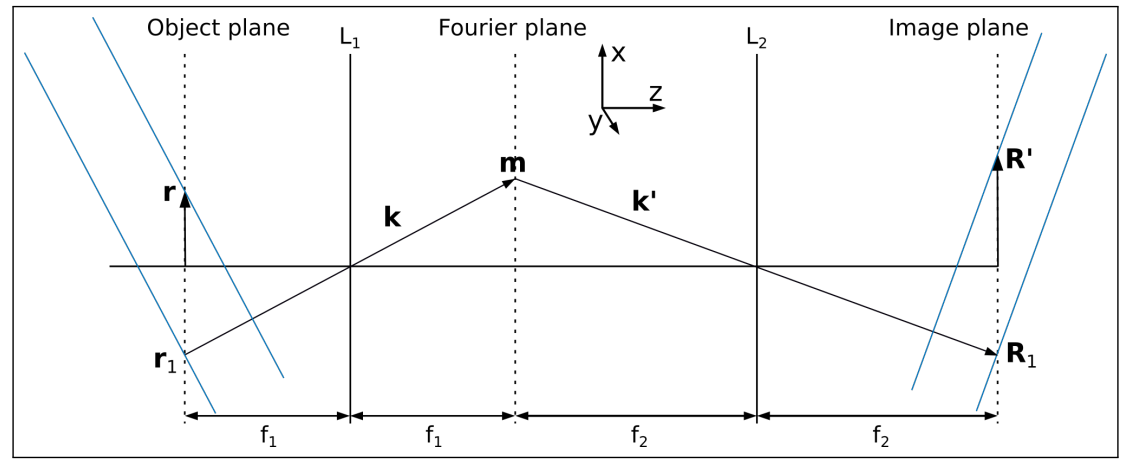

Fig. 6. Declaration of variables: $\mathbf{r}$ coordinate at the object plane; $\mathbf{r}_{1}$ coordinates of the intersection of the object plane and the center ray $\left(\mathrm{L}_{1}\right)$ with propagation direction $\mathbf{k} ; \mathbf{R}^{\prime}$ coordinate of the image plane; $\mathbf{R}_{1}$ coordinates of the intersection of the image plane and the center ray $\left(\mathrm{L}_{2}\right)$ with propagation direction $\mathbf{k} ; \mathbf{m}$ coordinates of the intersection of the Fourier plane and the center ray $\left(\mathrm{L}_{1}\right)$ with propagation direction $\mathbf{k}$.

\section{Appendix B - analysis of the third order susceptibility tensor}

In this appendix we detail some properties of $\overleftrightarrow{\chi}_{a S}^{(3)}$. In general, the nonlinear susceptibility comprises 81 elements. For an isotropic medium only 21 elements are non-zero and they satisfy [49]:

$$
\begin{aligned}
& \chi_{1111}=\chi_{2222}=\chi_{3333} \\
& \chi_{1122}=\chi_{1133}=\chi_{2211}=\chi_{2233}=\chi_{3311}=\chi_{3322} \\
& \chi_{1212}=\chi_{1313}=\chi_{2323}=\chi_{2121}=\chi_{3131}=\chi_{3232} \\
& \chi_{1221}=\chi_{1331}=\chi_{2112}=\chi_{2332}=\chi_{3113}=\chi_{3223}
\end{aligned}
$$

where $\chi_{a S, p, P, S}$ links the $a S$ component of the anti-Stokes polarization to the, $(p, P, S)$ components of the (pump, Probe, Stokes) fields and $(1,2,3)=(x, y, z)$. Thus, there are in total 4 independent elements, which are further linked by the relation:

$$
\chi_{1111}=\chi_{1122}+\chi_{1212}+\chi_{1221}
$$


In the experiment, we have placed an X-oriented polarization filter in front of the detector and use a low NA collection objective. Thus, we can assume that the image depends essentially on the $x$ component of the anti-Stokes polarization, which means that we do not probe the susceptibility components that start with 2 or 3 . In the Stokes scanning configuration, the pump beam impinging on the sample is $x$-polarized while the Stokes polarization may exhibit $(x, y, z)$ components. Yet, because of the tensor property, only the $\chi 1111$ is involved in the image formation. In the pump scanning configuration, the Stokes field is $x$-polarized while the polarization of the pump may vary. In this case, $\chi_{1111}, \chi_{1221}$ and $\chi_{1331}$, are involved in the image formation. In the synthetic experiments, we set $\chi_{1111}$ to 1 and $\chi_{1221}=\chi_{1331}$ to $1 / 3$.

\section{Appendix C - derivation of the gradient $g$}

The expression for the gradient $g$ is derived in [42] for any intensity imaging scheme. Here, we propose a less conceptual approach based on the variation of the cost functional when the scatterer density $N(\mathbf{r})$ is modified at $\mathbf{r}_{0}=\left(\mathbf{r}_{\| 0}, z_{0}\right)$. We introduce $\Delta N(\mathbf{r})=\delta N \delta\left(\mathbf{r}-\mathbf{r}_{0}\right)$ where $\delta N$ is complex and the variation of $N$. The gradient $g$ at point $\mathbf{r}_{0}$ is defined via,

$$
F(N+\Delta N)-F(N)=\operatorname{Re}\left[\delta N g^{*}\left(\mathbf{r}_{0}\right)\right]+o(\delta N) .
$$

We first derive the expression of the field variation when $N$ is changed to $N+\Delta N$, using Eq. (21), its linearity with respect to $N(r)$ and (12):

$$
\begin{aligned}
\Delta \mathbf{E}_{a S, l}\left(M \mathbf{R}_{\|}\right) & =\mathbf{E}_{a S, l}[N+\Delta N]\left(M \mathbf{R}_{\|}\right)-\mathbf{E}_{a S, l}[N]\left(M \mathbf{R}_{\|}\right) \\
& =\frac{i}{2} \frac{\delta N}{4 \pi^{2}} \int \mathrm{d} \mathbf{k}_{\|} e^{i \mathbf{k}_{\|} \cdot\left(\mathbf{R}_{\|}-\mathbf{r}_{\| 0}\right)-i \gamma z_{0}} \mathcal{H}\left(\mathbf{k}_{\|} \overleftrightarrow{\mathbf{Q}}\left(\mathbf{k}_{a S}^{\prime}, \mathbf{k}_{a S}\right) \overleftrightarrow{\mathbf{M}}\left(\mathbf{k}_{a S}\right) \mathbf{U}_{l}\left(\mathbf{r}_{0}\right)\right.
\end{aligned}
$$

where $\mathbf{U}_{l}\left(\mathbf{r}_{0}\right)=\overleftrightarrow{\chi}^{(3)^{\prime}} \mathbf{E}_{p, l}^{2}\left(\mathbf{r}_{0}\right) \mathbf{E}_{S, l}^{*}\left(\mathbf{r}_{0}\right)$ and we recall that $\mathbf{k}_{a S}=\mathbf{k}_{\|}+\gamma \mathbf{z}$ while $\mathbf{k}_{a S}^{\prime}=\mathbf{k}_{\|} / M+\gamma^{\prime} \mathbf{z}$ Then, we calculate the variation of the cost functional using Eq. (35),

$$
\begin{aligned}
F(N+\Delta N) & =\left.\left.W^{-1} \sum_{l} \int \mathrm{d}\left(M \mathbf{R}_{\|}\right)\left|I_{l}^{\mathrm{mes}}-\right| \mathbf{E}_{a S, l}[N+\Delta N]\right|^{2}\right|^{2} \\
& =W^{-1} \sum_{l} \int \mathrm{d}\left(M \mathbf{R}_{\|}\right)\left|I_{l}^{m e s}-\left(\mathbf{E}_{a S, l} \mathbf{E}_{a S, l}^{*}+2 \operatorname{Re}\left(\mathbf{E}_{a S, l}^{*} \Delta \mathbf{E}_{a S, l}\right)+\Delta \mathbf{E}_{a S, l} \Delta \mathbf{E}_{a S, l}^{*}\right)\right|^{2} \\
& =F(N)-4 W^{-1} \operatorname{Re} \sum_{l} \int \mathrm{d}\left(M \mathbf{R}_{\|}\right)\left[t_{l} \mathbf{E}_{a S, l}^{*} \cdot \Delta \mathbf{E}_{a S, l}\right]+o(\delta N)
\end{aligned}
$$

where $t_{l}=I_{l}^{\text {mes }}-\left|\mathbf{E}_{a S, l}[N]\right|^{2}$ is the residue at previous iteration and the dependence of $I_{l}^{\text {mes }}$, $\mathbf{E}_{a S, l}, \Delta \mathbf{E}_{a S, l}$ with respect to $M \mathbf{R}_{\|}$and $N$ is implicit. Introducing Eq. (35) in Eq. (36) yields,

$$
\begin{aligned}
F(N+\Delta N)-F(N)= & -4 W^{-1} \operatorname{Re}\left(\frac{i \delta N}{4 \pi^{2}} \sum_{l} \int \mathrm{d}\left(M \mathbf{R}_{\|}\right) \int \mathrm{d} \mathbf{k}_{\|} \mathcal{H}\left(\mathbf{k}_{\|}\right) e^{i \mathbf{k}_{\|} \cdot\left(\mathbf{R}_{\|}-\mathbf{r}_{\| 0}\right)-i \gamma z_{0}}\right. \\
& \left.t_{l} \mathbf{E}_{a S, l}^{*}\left(M \mathbf{R}_{\|}\right) \cdot \overleftrightarrow{\mathbf{Q}}\left(\mathbf{k}_{a S}^{\prime}, \mathbf{k}_{a S}\right) \overleftrightarrow{\mathbf{M}}\left(\mathbf{k}_{a S}\right) \mathbf{U}_{l}\left(\mathbf{r}_{0}\right)\right)+o(\delta N)
\end{aligned}
$$

which yields, after reordering the integrals and conjugating the expression,

$$
\begin{aligned}
& F(N+\Delta N)-F(N)=-4 W^{-1} \operatorname{Re}\left(\delta N^{*} \sum_{l} \int \mathrm{d} \mathbf{k}_{\|} e^{i \mathbf{k}_{\|} \cdot \mathbf{r}_{\| 0}} e^{i \gamma z_{0}} \mathcal{H}\left(\mathbf{k}_{\|}\right)\right. \\
& \left.\overleftrightarrow{\mathbf{Q}}\left(\mathbf{k}_{a S}^{\prime}, \mathbf{k}_{a S}\right) \overleftrightarrow{\mathbf{M}}\left(\mathbf{k}_{a S}\right) \mathbf{U}_{l}^{*}\left(\mathbf{r}_{0}\right) \cdot \frac{-i}{4 \pi^{2}} \int \mathrm{d}\left(M \mathbf{R}_{\|}\right) e^{-i \mathbf{k}_{\|} \cdot \mathbf{R}_{\|}} t_{l} \mathbf{E}_{a S, l}\right)^{*} \\
& =4 W^{-1} \operatorname{Re}\left(i \delta N^{*} \sum_{l}\right. \\
& \left.\mathbf{U}_{l}^{*}\left(\mathbf{r}_{0}\right) \cdot \mathcal{F}_{2 D}^{-1}\left\{e^{i \gamma z_{0}} \mathcal{H}\left(\mathbf{k}_{\|}\right) \overleftrightarrow{\mathbf{Q}}\left(\mathbf{k}_{a S}, \mathbf{k}_{a S}^{\prime}\right) \overleftrightarrow{\mathbf{M}}\left(\mathbf{k}_{a S}^{\prime}\right) \mathcal{F}_{2 D}\left[t_{l} \mathbf{E}_{a S, l}\right]\right\}\right)(38)
\end{aligned}
$$


where we have used the property $\mathbf{v} \cdot \overleftrightarrow{\mathbf{Q}}\left(\mathbf{k}_{a S}^{\prime}, \mathbf{k}_{a S}\right) \overleftrightarrow{\mathbf{M}}\left(\mathbf{k}_{a S}\right) \mathbf{U}=\mathbf{U} \cdot \overleftrightarrow{\mathbf{Q}}\left(\mathbf{k}_{a S}, \mathbf{k}_{a S}^{\prime}\right) \overleftrightarrow{\mathbf{M}}\left(\mathbf{k}_{a S}^{\prime}\right) \mathbf{v}$ and $\overleftrightarrow{\mathbf{Q}} \overleftrightarrow{\mathbf{M}}$ as well as $\mathcal{H}$ and $t_{l}$ are real. Comparing Eq. (34) and Eq. (38), one obtains the gradient,

$$
g\left(\mathbf{r}_{0}\right)=\frac{4 i}{W} \sum_{l} \mathbf{U}_{l}^{*}\left(\mathbf{r}_{0}\right) \cdot \mathcal{F}_{2 D}^{-1}\left\{e^{i \gamma z_{0}} \mathcal{H}\left(\mathbf{k}_{\|}\right) \overleftrightarrow{\mathbf{Q}}\left(\mathbf{k}_{a S}, \mathbf{k}_{a S}^{\prime}\right) \overleftrightarrow{\mathbf{M}}\left(\mathbf{k}_{a S}^{\prime}\right) \mathcal{F}_{2 D}\left[t_{l} \mathbf{E}_{a S, l}\right]\right\}\left(\mathbf{r}_{0}\right)
$$

\section{Appendix D - derivation of the scaling factor $\alpha$}

Once the search direction is established, the scaling factor $\alpha$ has to be optimized. We start from Eq. (34),

$$
F(N+\Delta N)=W^{-1} \sum_{l} \int\left|t_{l}-2 \operatorname{Re}\left(\mathbf{E}_{a S, l}^{*} \cdot \Delta \mathbf{E}_{a S, l}\right)-\Delta \mathbf{E}_{a S, l} \cdot \Delta \mathbf{E}_{a S, l}^{*}\right|^{2} \mathrm{~d}\left(M \mathbf{R}_{\|}\right) .
$$

We introduce the polynomial coefficients,

$$
\begin{aligned}
P_{l}^{00} & =\int\left|t_{l}\right|^{2} \mathrm{~d}\left(M \mathbf{R}_{\|}\right) \\
P_{l}^{11} & =\int\left|2 \operatorname{Re}\left(\mathbf{E}_{a S, l}^{*} \cdot \Delta \mathbf{E}_{a S, l}\right)\right|^{2} \mathrm{~d}\left(M \mathbf{R}_{\|}\right) \\
P_{l}^{22} & =\int\left|\Delta \mathbf{E}_{a S, l} \cdot \Delta \mathbf{E}_{a S, l}^{*}\right|^{2} \mathrm{~d}\left(M \mathbf{R}_{\|}\right) \\
P_{l}^{01} & =\int t_{l} 2 \operatorname{Re}\left(\mathbf{E}_{a S, l}^{*} \cdot \Delta \mathbf{E}_{a S, l}\right) \mathrm{d}\left(M \mathbf{R}_{\|}\right) \\
P_{l}^{02} & =\int t_{l} \Delta \mathbf{E}_{a S, l} \cdot \Delta \mathbf{E}_{a S, l}^{*} \mathrm{~d}\left(M \mathbf{R}_{\|}\right) \\
P_{l}^{12} & =\int 2 \operatorname{Re}\left(\mathbf{E}_{a S, l}^{*} \cdot \Delta \mathbf{E}_{a S, l}\right) \Delta \mathbf{E}_{a S, l} \cdot \Delta \mathbf{E}_{a S, l}^{*} \mathrm{~d}\left(M \mathbf{R}_{\|}\right)
\end{aligned}
$$

At the $n$-th iteration, the scatterer density is modified by $\Delta N_{n}=\alpha_{n} g_{n}$ so that Eq. (40) becomes,

$$
F\left(\alpha_{n}\right) \approx W^{-1} \sum_{l}\left(P_{l}^{00}+\alpha_{n}^{2} P_{l}^{11}+\alpha_{n}^{4} P_{l}^{22}-2 \alpha_{n} P_{l}^{01}-2 \alpha_{n}^{2} P_{l}^{02}+2 \alpha_{n}^{3} P_{l}^{12}\right)
$$

Then, $\alpha_{n}$ is determined so as to minimize the polynomial $F\left(\alpha_{n}\right)$. It was found that the speed of reconstruction can be improved by multiplying $\alpha_{n}$ with a factor $l$ to yield $\alpha_{\text {eff }}=l \alpha_{n}$. After each iteration $l$ is reduced or increased in dependence whether $F_{n-1} / F_{n}-1$ is larger or smaller than $4 \%$.

\section{Funding}

Centre National de la Recherche Scientifique (CNRS); Aix-Marseille University A*Midex (ANR11-IDEX-0001-02, A-M-AAP-ID-17-13-170228-15.22-Rigneault); ANR grants France Bio Imaging (ANR-10-INSB-04-01) and France Life Imaging (ANR-11-INSB-0006) infrastructure networks; Plan cancer INSERM (PC201508, 18CP128-00).

\section{References}

1. A. Zumbusch, G. R. Holtom, and X. S. Xie, “Three-dimensional vibrational imaging by coherent anti-stokes raman scattering," Phys. Rev. Lett. 82, 4142-4145 (1999).

2. J.-X. Cheng and X. S. Xie, eds., Coherent Raman Scattering Microscopy (Series in Cellular and Clinical Imaging) (Chemical Rubber Company, 2013), 1st ed.

3. H. Rigneault and P. Berto, "Tutorial: Coherent raman light matter interaction processes," APL Photonics 3, 091101 (2018). 
4. W. Min, C. W. Freudiger, S. Lu, and X. S. Xie, "Coherent nonlinear optical imaging: Beyond fluorescence microscopy," Annu. Rev. Phys. Chem. 62, 507-530 (2011).

5. J. P. R. Day, K. F. Domke, G. Rago, H. Kano, H. Hamaguchi, E. M. Vartiainen, and M. Bonn, "Quantitative coherent anti-stokes raman scattering (CARS) microscopy," J. Phys. Chem. B 115, 7713-7725 (2011).

6. M. T. Cicerone and C. H. Camp, "Histological coherent raman imaging: a prognostic review," Analyst 143, 33-59 (2018).

7. T. W. Bocklitz, F. S. Salah, N. Vogler, S. Heuke, O. Chernavskaia, C. Schmidt, M. J. Waldner, F. R. Greten, R. Bräuer, M. Schmitt, A. Stallmach, I. Petersen, and J. Popp, "Pseudo-HE images derived from CARS/TPEF/SHG multimodal imaging in combination with raman-spectroscopy as a pathological screening tool," BMC Cancer 16, 534 (2016).

8. O. Chernavskaia, S. Heuke, M. Vieth, O. Friedrich, S. Schürmann, R. Atreya, A. Stallmach, M. F. Neurath, M. Waldner, I. Petersen, M. Schmitt, T. Bocklitz, and J. Popp, "Beyond endoscopic assessment in inflammatory bowel disease: real-time histology of disease activity by non-linear multimodal imaging," Sci. Rep. 6, 29239 (2016).

9. S. Heuke, O. Chernavskaia, T. Bocklitz, F. B. Legesse, T. Meyer, D. Akimov, O. Dirsch, G. Ernst, F. von Eggeling, I. Petersen, O. Guntinas-Lichius, M. Schmitt, and J. Popp, "Multimodal nonlinear microscopy of head and neck carcinoma - toward surgery assisting frozen section analysis," Head Neck 38, 1545-1552 (2016).

10. J. Zheng, D. Akimov, S. Heuke, M. Schmitt, B. Yao, T. Ye, M. Lei, P. Gao, and J. Popp, "Vibrational phase imaging in wide-field CARS for nonresonant background suppression," Opt. Express 23, 10756 (2015).

11. P. Berto, A. Jesacher, C. Roider, S. Monneret, H. Rigneault, and M. Ritsch-Marte, "Wide-field vibrational phase imaging in an extremely folded box-CARS geometry," Opt. Lett. 38, 709 (2013).

12. P. Berto, D. Gachet, P. Bon, S. Monneret, and H. Rigneault, "Wide-field vibrational phase imaging," Phys. Rev. Lett. 109 (2012).

13. Y. Shen, J. Wang, K. Wang, A. V. Sokolov, and M. O. Scully, "Wide-field coherent anti-stokes raman scattering microscopy based on picosecond supercontinuum source," APL Photonics 3, 116104 (2018).

14. C. Heinrich, S. Bernet, and M. Ritsch-Marte, "Wide-field coherent anti-stokes raman scattering microscopy," Appl. Phys. Lett. 84, 816-818 (2004).

15. C. Heinrich, C. Meusburger, S. Bernet, and M. Ritsch-Marte, "CARS microscopy in a wide-field geometry with nanosecond pulses," J. Raman Spectrosc. 37, 675-679 (2006).

16. C. Heinrich, A. Hofer, S. Bernet, and M. Ritsch-Marte, "Coherent anti-stokes raman scattering microscopy with dynamic speckle illumination,” New J. Phys. 10, 023029 (2008).

17. A. Jesacher, C. Roider, S. Khan, G. Thalhammer, S. Bernet, and M. Ritsch-Marte, "Contrast enhancement in widefield CARS microscopy by tailored phase matching using a spatial light modulator," Opt. Lett. 36, 2245 (2011).

18. I. Toytman, K. Cohn, T. Smith, D. Simanovskii, and D. Palanker, "Wide-field coherent anti-stokes raman scattering microscopy with non-phase-matching illumination," Opt. Lett. 32, 1941 (2007).

19. I. Toytman, D. Simanovskii, and D. Palanker, "On illumination schemes for wide-field CARS microscopy," Opt. Express 17, 7339 (2009).

20. K. Wicker and R. Heintzmann, "Resolving a misconception about structured illumination,” Nat. Photonics 8, 342-344 (2014).

21. M. Lei, "Video-rate wide-field coherent anti-stokes raman scattering microscopy with collinear nonphase-matching illumination," J. Biomed. Opt. 16, 021102 (2011).

22. K. M. Hajek, B. Littleton, D. Turk, T. J. McIntyre, and H. Rubinsztein-Dunlop, "A method for achieving super-resolved widefield cars microscopy,” Opt. Express 18, 19263 (2010).

23. K. Shi, P. S. Edwards, J. Hu, Q. Xu, Y. Wang, D. Psaltis, and Z. Liu, "Holographic coherent anti-stokes raman scattering bio-imaging," Biomed. Opt. Express 3, 1744 (2012).

24. A. S. Duarte, C. Schnedermann, and P. Kukura, "Wide-field detected fourier transform CARS microscopy," Sci. Rep. 6, 37516 (2016).

25. G. Zheng, R. Horstmeyer, and C. Yang, "Wide-field, high-resolution fourier ptychographic microscopy," Nat. Photonics 7, 739-745 (2013).

26. J.-X. Cheng, A. Volkmer, and X. S. Xie, "Theoretical and experimental characterization of coherent anti-stokes raman scattering microscopy,” J. Opt. Soc. Am. B 19, 1363 (2002).

27. N. Streibl, "Three-dimensional imaging by a microscope," J. Opt. Soc. Am. A 2, 121 (1985)

28. S. Heuke, F. B. Legesse, A. Lorenz, T. Pascher, D. Akimov, M. Jäger, M. Schmitt, and J. Popp, "Fiber-based dual-focus time-demultiplexed second harmonic generation microscopy,” Opt. Lett. 40, 2505 (2015).

29. M. Schrader, S. W. Hell, and H. T. M. van der Voort, "Three-dimensional super-resolution with a 4pi-confocal microscope using image restoration,” J. Appl. Phys. 84, 4033-4042 (1998).

30. O. Haeberlé, K. Belkebir, H. Giovaninni, and A. Sentenac, "Tomographic diffractive microscopy: basics, techniques and perspectives," J. Mod. Opt. 57, 686-699 (2010).

31. S. Khadir, P. C. Chaumet, G. Baffou, and A. Sentenac, "Quantitative model of the image of a radiating dipole through a microscope," J. Opt. Soc. Am. A 36, 478 (2019).

32. D. Gachet, N. Sandeau, and H. Rigneault, "Influence of the raman depolarisation ratio on far-field radiation patterns in coherent anti-stokes raman scattering (CARS) microscopy," J. Eur. Opt. Soc. Rapid Publ. 1 (2006).

33. L. Novotny and B. Hecht, "Preface," in Principles of Nano-Optics, (Cambridge University Press, 2006), pp. xv-xviii.

34. D. A. Smunev, P. C. Chaumet, and M. A. Yurkin, "Rectangular dipoles in the discrete dipole approximation," J. Quant. Spectrosc. Radiat. Transf. 156, 67-79 (2015). 
Optics EXPRESS

35. M. J. Huttunen, A. Abbas, J. Upham, and R. W. Boyd, "Label-free super-resolution with coherent nonlinear structured-illumination microscopy,” J. Opt. 19, 085504 (2017).

36. Z. Wen, C. Yang, X. Liu, and S. Marchesini, "Alternating direction methods for classical and ptychographic phase retrieval," Inverse Probl. 28, 115010 (2012).

37. L. Bian, J. Suo, J. Chung, X. Ou, C. Yang, F. Chen, and Q. Dai, "Fourier ptychographic reconstruction using poisson maximum likelihood and truncated wirtinger gradient," Sci. Rep. 6, 27384 (2016).

38. A. P. Konijnenberg, W. M. J. Coene, and H. P. Urbach, "Model-independent noise-robust extension of ptychography," Opt. Express 26, 5857 (2018).

39. C. Zuo, J. Sun, and Q. Chen, “Adaptive step-size strategy for noise-robust fourier ptychographic microscopy,” Opt. Express 24, 20724 (2016).

40. X. Ou, G. Zheng, and C. Yang, "Embedded pupil function recovery for fourier ptychographic microscopy," Opt. Express 22, 4960 (2014).

41. L. Tian and L. Waller, "3d intensity and phase imaging from light field measurements in an LED array microscope," Optica 2, 104 (2015).

42. K. Unger, T. Zhang, P. C. Chaumet, A. Sentenac, and K. Belkebir, "Linearized inversion methods for three-dimensional electromagnetic imaging in the multiple scattering regime," J. Mod. Opt. 65, 1787-1792 (2018).

43. E. Polak and G. Ribière, "Note sur la convergence de méthodes directions conjuguées," Revue française d'informatique et de recherche opérationelle 3, 35-43 (2017).

44. D. B. Murphy and M. W. Davidson, Fundamentals of Light Microscopy and Electronic Imaging (John Wiley \& Sons, Inc., 2012).

45. R. Horstmeyer, R. Heintzmann, G. Popescu, L. Waller, and C. Yang, "Standardizing the resolution claims for coherent microscopy," Nat. Photonics 10, 68-71 (2016).

46. G. Eckhardt, R. W. Hellwarth, F. J. McClung, S. E. Schwarz, D. Weiner, and E. J. Woodbury, "Stimulated raman scattering from organic liquids," Phys. Rev. Lett. 9, 455-457 (1962).

47. S. Heuke, B. Sarri, X. Audier, and H. Rigneault, "Simultaneous dual-channel stimulated raman scattering microscopy demultiplexed at distinct modulation frequencies," Opt. Lett. 43, 3582 (2018).

48. J. Chung, H. Lu, X. Ou, H. Zhou, and C. Yang, "Wide-field fourier ptychographic microscopy using laser illumination source," Biomed. Opt. Express 7, 4787 (2016).

49. R. W. Boyd, Nonlinear Optics (Academic Press, 2003). 